DATA PROCESSING IN THREE-DIMENSIONAL TEXTURE ANALYSIS

JERZY JURA and JAN POSPIECH

Polish Academy of Sciences, Institute of Metal Research Cracow, Poland

(Received March 8, 1976)

Abstract: To the background of purely computative problems of three-dimensional texture analysis, the characteristics of an extensive system of computer subroutines, which allow certain standard calculations to be carried out, are presented. These subroutines are written in FORTRAN, and are easily adapted to any computer possessing a FORTRAN compiler.

\title{
INTRODUCTION
}

The implementation of three-dimensional analysis techniques involves a great number of calculations. Their execution is feasible only in a computer by means of appropriate programs. In these calculations two stages can be distinguished: processing of experimental data, and texture analysis. First, the experimental data, in the form of pole figures or the results of single orientation measurements, are processed into coefficients $C_{L}^{\mu \nu}$ of the series expansion of the orientation distribution function (ODF). This stage can be considered as an extension of the measuring equipment's job. The computer processing the data may be on line with the measuring equipment or the data may be recorded on tape.

The coefficients $C_{L}^{\mu \nu}$ hold all of the information about the texture of the examined material and therefore they make up the basis for the second state, in which computative operations linked directly with the problem 
on hand are carried out. In order to analyze and describe the texture the ODF are computed. It is then possible to determine the quantitative shares of the various texture components and to establish the relationship between them. Any pole figure and any inverse pole figure

can be determined. The coefficients $C_{L}^{\mu \nu}$ allow texture transformations to be carried out with a given model of, say, a phase transformation, recrystallization or deformation. Three-dimensional analysis can be used to verify such models quantitatively. Together with computer simulation techniques, three-dimensional texture analysis makes it possible to find strict answers (in quantitative terms) to many questions relating to the behaviour of a polydrystalline material during various processes and the anisotropy of its properties.

The broad scope of three-dimensional analysis application induces continuous development of computer programs by which texture studies are implemented. The features of the various programs have been described in many publications. ${ }^{-4}$ Paper (3) also gives the source listing of the system of subroutines (in FORTRAN) which realize standard computations based on symmetrical pole figures measured over the full range for substances having cubic crystal lattices. Within the last couple of years a number of new procedures have been added to this system. In what follows the specifications of this extended system against the background of the computative problems of three-dimensional texture analysis are outlined.

\section{Characteristics of Calculations}

In the two stages mentioned above it is possible to distinguish certain characteristic computative problems. The experimental-data processing stage includes:

- preliminary processing of the data acquired by measurements, and

- standard calculations for determining the coefficients $C_{L}^{\mu \nu}$

The texture analysis stage comprises:

- the calculation and description of the distribution functions, and

- research of the models of texture transformations and an analysis of the effect of texture on the anisotropy of material properties. 


\subsection{Preliminary Processing of Experimental Data}

There are in general two kinds of data: pole figures and the results of measurements of single orientations. The experimental material acquired from the pole figure measurement is very diversified, depending on the equipment used and on the symmetry of the measured figures. Also, measurements sometimes reveal the existence of coincidence of reflexes from two or more planes.

If the pole figure can be measured by the backreflection technique at least up to an angle $\alpha=70-75^{\circ}$, or by the transmission technique at least starting from $\alpha=30-35^{\circ}$, it is then advantageous to process the results acquired by one measuring technique only, the socalled incomplete pole figures. The number of measured pole figures should not be smaller than four, however, because although calculation of the coefficients

$C_{L}^{\mu \nu}$ then becomes more complicated, ${ }^{5-7}$ the results are just as accurate or even better than when complete pole figures in lesser numbers are used.

When the measurement range does not reach the values of angle just mentioned, then as a rule it is necessary to utilize both measuring techniques. Use is then made of complete pole figure processing. In this case, however, preparation of the samples and the measurement itself are more laborious. Apart from this, efforts to make the results of both techniques compatible may introduce new errors.

The input data for the processing system are several pole figures usually measured in intervals $\Delta \alpha=\Delta \beta=5^{\circ}$, and the values of the background and corrective data. In the first computative stage the measured figures are corrected for background, absorption and other effects. In the case of complete pole figures it is necessary to render compatible the parts acquired by the back-reflection and transmission techniques. The methods of correcting the data vary enormously. Usually, each series of measurements requires an individual approach and special computative procedures have to be prepared.

The results of measurements of single orientations are processed into Euler angles. A Gaussian distribution spread is usually ascribed to each orientation. The half-width of the distribution is chosen appropriately to the estimated error of a single-orientation measurement. Thanks to this, the form of the data approximates the actual experimental conditions better and, at the same time, the error involved with the truncation of the series is diminished. 
1.2. Determining the Values of Coefficients $C_{L}^{\mu \nu}$

The values of the coefficients $C_{L}^{\mu \nu}$ are calculated on the basis of several corrected pole figures or several hundred single orientations measured or determined by computer simulation. This part of the calculations also gives an estimate of the dispersion of the determined $C_{L}^{\mu \nu}$, together with the error associated with series truncation.

To compute the values of $C_{L}^{\mu \nu}$, use is made of the mean square approximation of the experimental data by employing a series of symmetrical spherical functions with unknown coefficients $C_{L} \mu \nu$.

When the data are in the form of pole figures, the approximating formula has the general form

$$
\int_{D_{h}} \int_{D_{Y}} w_{1}(h) w_{2}(y)\left[A(h, y)-\sum_{L, \mu \nu}^{L_{\max }} \frac{4 \pi}{2 L+1} C_{L}^{\mu \nu} \dot{k}_{L}^{\mu}(h) \dot{k}_{L}^{\nu}(y)\right]^{2} d y d h=\min
$$

where $A(h, y)$ is known as the general axis distribution function ${ }^{5}$ [pole figure $\left.P_{h_{i}}(y)=A\left(h_{i}, y\right)\right], D_{h}$ and $D_{y}$ denote the regions in which the function $A(h, y)$ has been determined, and $w_{1}(y), w_{2}(h)$ are the so-called weight functions.

When the results of measurements of single orientations are used as the input data, we have the expression

$$
\int_{G} w_{3}(g)\left[f(g)-\sum_{L, \mu \nu}^{L_{\max }} C_{L}^{\mu \nu} \dot{\mathrm{T}}_{L}^{\mu \nu}(g)\right]^{2} d g=\min
$$

where $f(g)$ denotes the ODF, $G$ is the region in which the measurement of orientation $g$ was performed, and $w_{3}(g)$ is weight function.

In practice, when the coefficients $C_{L}^{\mu \nu}$ minimalizing the expressions (1) and (2) are being sought, use is made of one of the algorithms of numerical integration, say, by the trapezoid method, or integration is replaced by simple summation. It is important to take account of the accuracy of the experimental data (i.e. their variance). To this aim, the functions $w_{1}(h), w_{2}(y)$ and $w_{3}(g)$ have been introduced. 
When the input data are incomplete pole figures measured for materials of symmetry lower than cubic, the problem of determining the values of the coefficients $C_{L}^{\mu \nu}$ is brought down to solving a system of equations containing several hundred unknowns. Prime importance is the focused on the question of the conditioning of the system's coefficient matrix and on the lack of stability, i.e., the sensitivity to errors of algebraic rounding offs. It is then essential to make the right choice of numerical procedure for solving the system of equations, and the accuracy of the calculations, related with computer word length, should be appropriate. The latter can be improved by carrying out calculations with a double precision and by using a special kind of program compilation which decreases the effect of rounding off values. Such difficulties do not arise when calculations involve incomplete pole figures of materials having cubic lattices and when complete pole figures are available.

To this stage we may also include the determination of coefficients $c^{\mu \nu}$ for model distributions obtained, for instance, by superimposing Gaussian distributions about several ideal orientations.

\subsection{Calculation and Description of Distribution Functions}

In the second stage the coefficients $C_{L}^{\mu \nu}$ are used to elucidate specific problems associated with texture analysis. First of all, the ODF are determined in Euler angle space or in the space formed by the parameters of the axis and the angle of rotation. ${ }^{8}$ The latter of these spaces is particularly convenient for depicting the relationship between the orientations of the crystallites of the two phases. It is also possible to determine the pole figures for a definite lattice plane and the inverse pole figures for a definite direction of the sample. If the computer is equipped with a plotter, the results of calculations can be presented in the form of ready plots of the ODF, pole figures and inverse figures.9 Very important, but hitherto inadequately developed, are the methods of estimating the dispersion and confidence intervals of the distribution functions. A full analysis of errors should take account of both the errors of the experimental data and the errors stemming from the use of approximating methods; it should also be remembered that errors caused by rounding off algebraic expressions may arise when multicomponent sums of series defining the values of functions are being calculated. 
When a texture analysis is being made, the ODF may be treated in approximation as the superposition of distribution functions of a defined shape, say, Gaussian. The maxima of the distributions are then assigned to points at which the ODF calculated on the basis of experimental data also attain their highest values. Thanks to this, it is possible to describe texture by means of several parameters which characterize the shares of the components in the texture and their spread. These are obtained by fitting the model function to the ODF. 10

1.4. Finding the Texture Transformation Model and Analyzing the Anisotropy of Properties

Three-dimensional analysis allows various geometrical models of texture transformation to be tested. Among other things, it was found to be useful when describing the phase transformations in accordance with Kurdyumov-Sachs model. 1 The development of texture in deformation of materials having a cubic lattice was examined with assumed Taylor and Sachs models.12-15 The computative methods of three-dimensional analysis are also used when describing the formation of a recrystallization texture. ${ }^{5}, 16$ Determination of the coefficients $C_{L}^{\mu \nu}$ also allows the texture to be linked up with the anisotropy of properties (in accordance with the assumed
model).

The division of the calculations involved in threedimensional analysis discussed here is illustrated by the block diagram in Figure 1.

\section{Sets of Tabuzar Values}

To carry out the calculations encountered in threedimensional analysis it is indispensible to possess an appropriate set of tabulated values required in each of the calculation stages. These values form a permanent file recorded on a disk or magnetic tape. This file is assembled by a special library program. First of all, the tabular values contain the Fourier coefficients of the generalized spherical functions $a_{L}^{\prime m n s}$ and the symmetry coefficients of the cubic spherical functions $\dot{\mathrm{B}}_{\mathrm{L}}^{\mathrm{m}} \mathrm{h}$ of very high accuracy and determined to an appropriately high order of $1_{\max } \cdot{ }^{17-20}$ The sets used in practice contain six-digit values of $a_{L}^{\prime m n s}$ and $\dot{B}_{L}^{m \mu}$ to the order $l_{\max }=34$ which, bearing in mind the unavoidable 


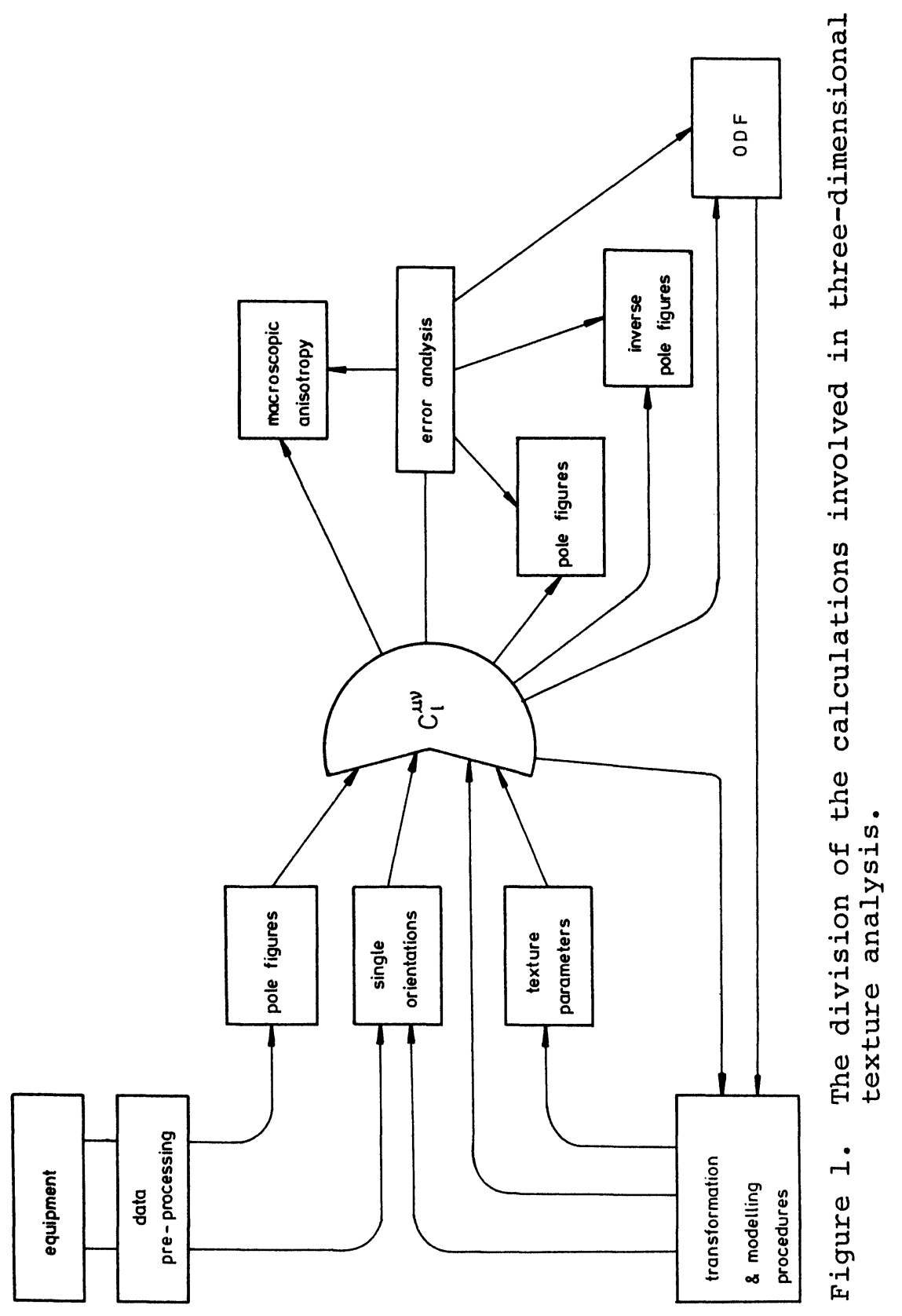


errors of the measured data, can be accepted to be quite adequate.

It is advantageous also to tabulate the values of some symmetrical spherical functions $\dot{\mathrm{k}}_{L}^{\mu}\left(h_{i}\right)$ and $\dot{\mathrm{T}}_{L}^{\mu \nu}\left(g_{i}\right)$, and also the associated Legendre polynomials $\mathrm{P}_{1}^{\mathrm{m}}\left(\alpha_{i}\right)$.

Moreover, when working with incomplete pole figures it is convenient to have a table of the integrals

$$
\zeta_{L L}^{\nu L^{\prime}}\left(\alpha_{0}\right)=\int_{0}^{2 \pi} \int_{\alpha_{0}}^{\pi / 2} \dot{k}_{L}^{\nu}(\alpha, \beta) \dot{k}_{L}^{* \nu}(\alpha, \beta) \sin \alpha d \alpha d \beta
$$

determined for different values of $\alpha_{0}$.

3. System of subroutines

The enormous variety of the problems involved in three-dimensional texture analysis gives rise to the necessity of developing more and more new programs. It seems better not to build large universal programs, but instead work out short subroutines and procedures for solving single definite problems. A collection of such subroutines then forms a kind of data processing system. Short programs of an organizational character pick out the appropriate subroutines from the system when required, put them together and define the succession of their execution. The characteristic features of such a system will now be outlined.

\subsection{System structure}

The system consists of several tens of subroutines, each of which carries out the calculations involved in one of the standard problems described earlier. Apart from this, it contains subroutines which just organize the work to be done, i.e. the read-in of data and printout of results in a standard form, the choice of the input/output device to be used, etc.

Routines which calculate the tabular values and form the library files are auxiliary programs. The tabular values needed for the calculations are recorded on magnetic tape.

Table I gives the functions and names of the more important subroutines, and some of them have been described in detail elsewhere. ${ }^{2}, 3$ The utilization of the peripheral devices in such calculations is shown in Figure 2. 


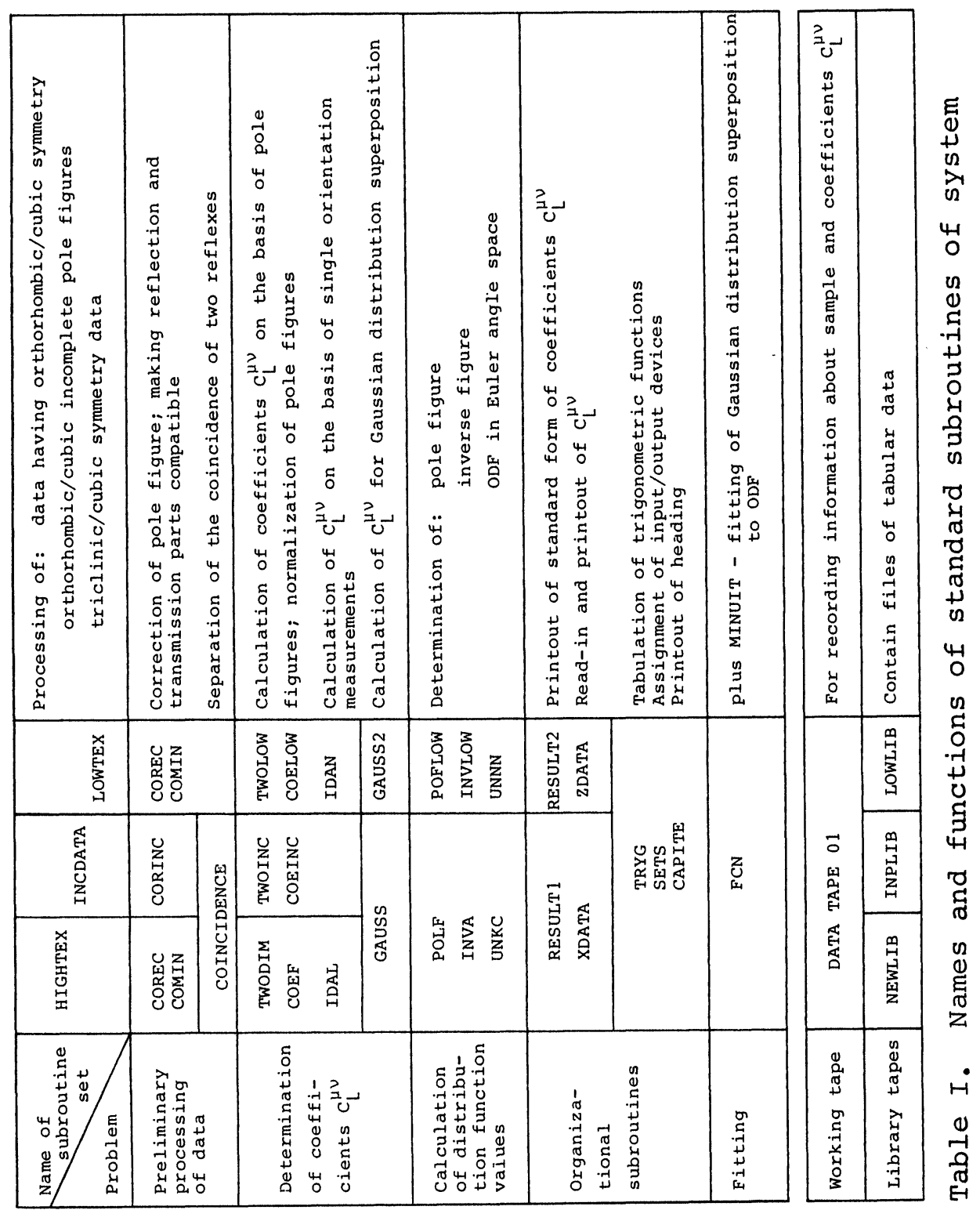




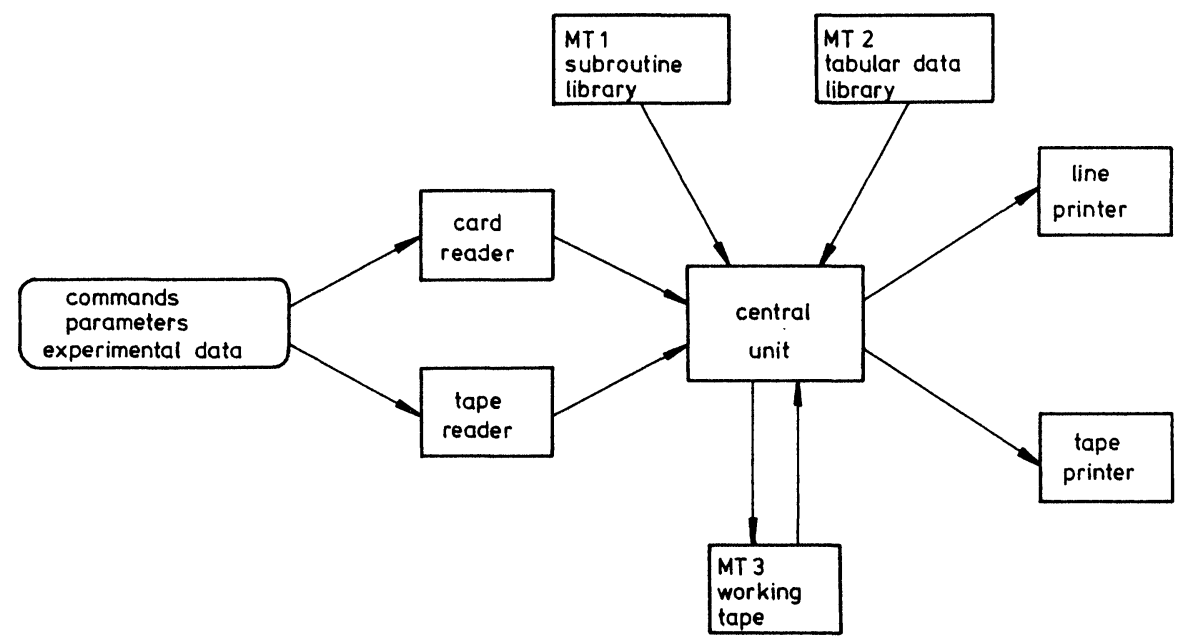

FIGURE 2. The utilization of the peripheral devices by the programs.

\subsection{Range of Calculations}

The system enables processing of typical experimental data, i.e. complete and incomplete pole figures measured solely by either the back-reflection or transmission technique, and the results of measurements of single orientations. It is also possible to process pole figures in which coincidences of refleces from two planes are seen to occur.

The system is intended to work with data from materials having crystal lattices of cubic to orthorhombic symmetry. The symmetry of the sample is arbitrary. Incomplete pole figures make an exception; their symmetry cannot be lower than orthorhombic. The operational range of the system is illustrated by Table II. The symmetries to which the standard subroutines of the system apply are underlined.

Routine calculations include determination of the coefficients $C_{L}^{\mu \nu}$ together with an estimate of their dispersion and the determination of the basic distribution functions, viz., ODF, pole figures and inverse pole figures. There is also a subroutine which computes the coefficients $C_{L}^{\mu \nu}$ for an ODF representing a superposition of Gaussian distributions, and a procedure fits this superposition to the ODF acquired on the basis of measured data. This procedure cooperates with the program MINUIT.21 (This program consists of a number of subroutines for determining the minimum of miltivariable functions.) The fit is usually done by the Monte Carlo and Simplex methods. The result is a set of values of 


\begin{tabular}{|l|l|l|}
\hline \multicolumn{2}{|c|}{$\begin{array}{c}\text { Symmetry in } \\
\text { Symmetry } \\
\text { in sample } \\
\text { system } \\
\text { system }\end{array}$} & $\begin{array}{l}\text { cubic, hexagonal, tetragonal, } \\
\text { orthorhombic }\end{array}$ \\
\hline \multirow{2}{*}{ Pole } & Complete & $\begin{array}{l}\text { triclinic, orthorhombic, } \\
\text { cylindrical }\end{array}$ \\
\cline { 2 - 3 } & Incomplete & orthorhombic, cylindrical \\
\hline $\begin{array}{l}\text { Measurement of } \\
\text { single orientations }\end{array}$ & $\begin{array}{l}\text { triclinic, orthorhombic, } \\
\text { cylindrical }\end{array}$ \\
\hline
\end{tabular}

Table II

the shares of the various texture components and the parameters characterizing the spread about the ideal positions, being a synthetic characteristic of the ODF.

\subsection{Brief Technical Specification}

All subroutines and programs of the system are written in FORTRAN. They are easily adapted to work with any kind of computer provided with a FORTRAN compiler. The standard programs require at most $30 \mathrm{kilo-}$ words of the central memory, the exact value being dependent on the type of computer used.

With a CDD 6400 series computer, the execution time of a program involving the determination of the coefficients $C_{L}^{\mu \nu}$ on the basis of complete pole figures, or the calculation of a pole figure or inverse figure is a dozen-odd seconds; whereas the determination of $C_{L}^{\mu \nu}$ on the basis of incomplete pole figures or measured values of single orientations or the calculation of an ODF or fitting it to experimental data takes several minutes. Tapes with tabular values contain the coefficients: $a_{L}^{\prime m n s}, a_{L}^{\prime n s}$ and $\dot{B}_{I}^{m \mu}$. In addition, some values of the functions $\mathrm{P}_{1}^{\mathrm{n}}\left(\alpha_{0}\right), \dot{\mathrm{k}}_{L}^{\mu}\left(\mathrm{h}_{i}\right)$ and $\dot{\mathrm{T}}_{L}^{\mu \nu}\left(g_{i}\right)$ and the integral $\zeta_{L L}^{U L^{\prime}}\left(\alpha_{0}\right)$ [formula (3)] are also tabulated. These. 
quantities are recorded for values of $1_{\max }$ up to the order of 34. The integrals of the spherical functions are computed up to $I_{\max }=22$ for radial angle values of $5^{\circ}, 10^{\circ}, 15^{\circ}, \ldots, 90^{\circ}$. Such a set was quite sufficient for the problems hitherto considered. The structure of the library file NEWLIB of Table I has been described in detail in papers (2) and (3).

Summary

The development of the methods and applications of three-dimensional texture analysis is closely associated with the continuous improvement of the mathematical apparatus used. The formation of optimal sets of subroutines which comprise a number of basic computative problems permit large quantities of experimental data to be processed and analyzed quickly.

The described system makes it possible to process almost all kinds of measured data concerning texture encountered in practice. It cannot cope with monoclinic and triclinic crystalline symmetry, but this has no practical bearing at all in metallography.

\section{REFERENCES}

1. H. J. Bunge, Kristall und Technik, 6, 429 (1971).

2. J. Jura, J. Pospiech and H. J. Bungē, Texture, 1 , 201 (1974).

3. J. Jura, J. Pospiech and H. J. Bunge, Prace Komisji Metalurgicano-odlewniczej (Proceedings of MetalZurgical-Casting Commission), Polish Academy of Sciences, Krakow Division (in press).

4. A. Clement and G. Durant, J.Appl. Cryst., 8, 589 (1975).

5. H. J. Bunge, Mathematische Methoden der Texturanalyse, Verlag-Berlin, 1969.

6. J. Pospiech and J. Jura, Z. Metallkunde, 65, 324 $(1974)$.

7. P. R. Morris, Advances in X-Ray Analysis, 18, 514 (1975).

8. J. Pospiech, Kristall und Technik, 7, 1057 (1972).

9. K. W. Bergman, W. Brunn and R. Kern, Texture, (in press).

10. W. Truszkowski, J. Pospiech, J. Jura and B. Major, Proceedings of "ze colloque europeen sur les textures," Pont á Mousson, 1973, pp. 235-258.

11. J. Pospiech, J. Jura and A. Maciosowski, ibid., pp. 117-136.

12. H. J. Bunge, Kristall und Technik, 5, 145 (1970). 
13. H. J. Bunge and T. Leffers, Scripta Met., $\underline{5}, 143$ (1971).

14. J. S. Kallend and G. J. Davies, Phil. Mag., 25, 471 (1972).

15. J. Jura and K. Wierzbanowski, Arch. Hutn., (in press).

16. V. Schmidt, K. Lücke and J. Pospiech, Proceedings of "Fourth European Colloquium on Textures," Cambridge, 1975 (in press).

17. H. J. Bunge, Kristall und Technik, 8, 939 (1974).

18. J. Pospiech and J. Jura, ibid., 10, 783 (1975).

19. P. R. Morris, Texture, 1, 57 (1975).

20. C. Esling, E. Bechler and R. Baro, Proceedings of "Tenth International Congress of Crystallography," August, 1975 (in press).

21. CERN Program Library programme D506. 